\title{
A reflective TWTA predistortion linearizer for Ka-band application
}

\author{
Wang Yongfei \\ Dept. Electromagnetic Wave and Antenna \\ Information Science and Technology Institute \\ Zhengzhou, China \\ lavender0303@yahoo.cn \\ Zhou Dongfang \\ Dept. Electromagnetic Wave and Antenna \\ Information Science and Technology Institute \\ Zhengzhou, China \\ zhdfxd@yahoo.cn
}

\author{
Hu Qing \\ Electronic Engineering Institute \\ Hefei, China \\ Doctor3elong@sina.com
}

\section{Zhao Ying}

Dept. Electromagnetic Wave and Antenna Information Science and Technology Institute Zhengzhou, China zhying2005@163.com

\section{BASIC PRINCIPLE AND SIMULATION}

\begin{abstract}
A predistortion linearizer used for Ka-band travelling wave tube amplifiers (TWTAs) was proposed. Based on analysis of nonlinear transfer characteristics of TWTA using a power series, the inverse nonlinear distortion characteristics of predistorter which was also the model of predistortion linearizer was proposed. A reflective structure was used to realize the linearizer, it's composed of anti-parallel schottky diodes, quadrature hybrid, matching networks seen from the diodes. The Carrier-to-Intermodulation(C/I) of Kaband TWTA was improved a maximum of $19 \mathrm{~dB}$ by proposed predistortion linearizer.
\end{abstract}

Keywords-predistortion linearizer; nonlinear transfer characteristics; Carrier-to-Intermodulation; anti-parallel schottky diodes

\section{INTRODUCTION}

The rapid growth of information to be exchanged has been calling for highly linear power amplifiers (PAs). High linearity is required for the spectrally efficient transmission of information. Traveling-wave-tube amplifiers (TWTAs), comparing with solid-state power amplifiers (SSPAs)[1] which are both common PAs at microwave frequency, have the advantages of high gain, high output power and good radioresistance. However, when working near the saturation region, the linearity degrades rapidly and the cause severe amplitude (AM-to-AM) and phase (AM-to-PM) distortion [2]. Linearizers are needed to compensate for the distortion. The linearization techniques include feedback, feedfoward and predistortion[2 9]. Considering its application to satellite communication, this paper utilizes a predistortion linearizer which is simple, small and stable. The predistortion linearizer here is composed of antiparallel schottky diodes together with a bias circuit, 90 degree hybrid as well as DC block.

\section{A. nonlinearity of TWTAs}

When excitating a TWTA working at its nonlinear region, the output spectrums contain the original signal as well as some undesired new frequencies. This is called nonlinear distortion, which is produced by a loss of linearity. The input-output transfer response of a TWT amplifier can be represented by a power series

$$
V_{\text {out } T}(t)=k_{1} V_{i T}(t)+k_{2} V_{i T}^{2}(t)+k_{3} V_{i T}^{3}(t)+\cdots=\sum_{n=1}^{\infty} k_{n} V_{i T}^{n}(t)
$$

where $V_{\text {iT }}(t)$ is the input signal, $V_{\text {out }}(t)$ is the output signal and $k_{n}$ the amplitude of $V_{i T}^{n}(t)$. When the TWTA works at its linear region, $k_{n}=0, n \geq 2$, otherwise, $k_{n}$ is incompletely equal to 0 . In case of weak nonlinearity, the first three terms are enough to characterize output signal.

When a single-carrier signal, e.g. a cosine wave signal, is substituted into this expression, in which $V_{i T}(t)=A \cos \omega_{1} t$, the original cosine wave as well as its harmonic distortion products will be contained in the output waveform. Unless the bandwidth is greater enough than an octave or more, these harmonics can be filtered by an extra band pass filter. However, when excited by a signal with more carriers, e.g. $V_{i T}(t)=A\left(\cos \omega_{1} t+\cos \omega_{2} t\right) \quad\left|\omega_{1}-\omega_{2}\right|<\omega_{1}, \omega_{2}$, intermodulation-distortion products are not neglectable. Then

$$
\begin{aligned}
& V_{\text {out }}(t)=k_{1} A\left(\cos \omega_{1} t+\cos \omega_{2} t\right)+k_{2} A^{2}\left(\cos \omega_{1} t+\cos \omega_{2} t\right)^{2} \\
& +k_{3} A^{3}\left(\cos \omega_{1} t+\cos \omega_{2} t\right)^{3}=\frac{1}{2} k_{2} A^{2}+\frac{1}{2} k_{2} A^{2}\left[\cos \left(\omega_{1}-\omega_{1}\right) t+\cos \left(\omega_{1}+\omega_{1}\right) t\right] \\
& +\left(k_{1} A+\frac{9}{4} k_{3} A^{3}\right)\left(\cos \omega_{1} t+\cos \omega_{2} t\right)+\frac{3}{4} k_{3} A^{3}\left[\cos \left(2 \omega_{2}+\omega_{1}\right) t+\cos \left(2 \omega_{1}+\omega_{2}\right) t\right] \\
& +\frac{3}{4} k_{3} A^{3}\left[\cos \left(2 \omega_{2}-\omega_{1}\right) t+\cos \left(2 \omega_{1}-\omega_{2}\right) t\right]+\frac{1}{4} k_{3} A^{3}\left(\cos 3 \omega_{1} t+\cos 3 \omega_{2} t\right)
\end{aligned}
$$

Parts of the intermodulation-distortion terms, $V_{\text {IMз }}$ is commonly known as third order intermodulation products, 
are so close to the carrier signals that could not be filtered and would affect the linearity.

\section{B. principle of predistortion}

Predistortion linearizers are widely used in microwave and satellite application due to that they are relatively simple and that they are independent to TWTAs that could be external components and reduce the design complexity. The predistortion linearizer(Fig. 1) can be thought as a black box whose I/O transfer characteristic is strictly opposite to that of the TWTA to be linearized. In other way, a predistorter is an IMD generator, the IMDs by which are equal in amplitude and 180 degree out of phase with the IMDs generated by the TWTA. So when cascaded together, the whole I/O transfer characteristic will satisfy the need for lossless transmission.

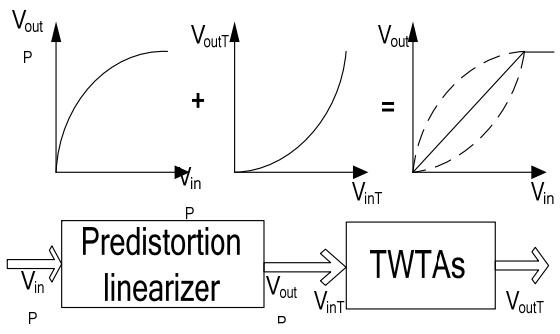

Fig. 1 schematic diagram of predistortion linearizer

Using a simple power series model to describe the predistortion linearizer, we get

$$
V_{\text {outP }}(t)=g_{1} V_{i P}(t)+g_{2} V_{i P}^{3}(t)(3)
$$

where $V_{i P}(t)$ and $V_{\text {outP }}(t)$ represent the input and output signal of the PD respectively, $g_{n}$ is the coefficient of $V_{\text {outP }}(t)$. Supposed that $V_{i P}(t)=B\left(\cos \omega_{1} t+\cos \omega_{2} t\right)$, signals contained in $V_{\text {outP }}(t)$ are the carrier signals $\left(\omega_{1}\right.$ 、 $\left.\omega_{2}\right)$, IMD signals( $\left.2 \omega_{2}-\omega_{1} 、 2 \omega_{1}-\omega_{2}\right)$. Substituting $V_{\text {outP }}(t)$ into $V_{\text {outT }}(t)$ as input signals of the TWTA, it can be taken as a pair of two-tone signals ( $\omega_{1} 、 \omega_{2}$ and $\left.2 \omega_{2}-\omega_{1} 、 2 \omega_{1}-\omega_{2}\right)$. Then output signals are the combination of two-tone signals $\left(\omega_{1} 、 \omega_{2}\right)$ together with their IMD signals ( $\left.2 \omega_{2}-\omega_{1} 、 2 \omega_{1}-\omega_{2}\right)$ and two-tone signals ( $\left.2 \omega_{2}-\omega_{1} 、 2 \omega_{1}-\omega_{2}\right)$ together with their IMD signals( $\left.5 \omega_{1}-4 \omega_{2} 、 5 \omega_{2}-4 \omega_{1}\right)$, signals at other frequencies could be filtered out. It can be proved that there would be no other signals generated at the frequencies of $2 \omega_{2}-\omega_{1}$ and $2 \omega_{1}-\omega_{2}$. So the third order IMD of the TWTA can be written as follows

$$
\begin{aligned}
& V_{I M 3}=\frac{3}{4} B^{3}\left(g_{3} k_{1}+g_{1}^{3} k_{3}+\left(\frac{9}{4}\right)^{3} k_{3} g_{3}^{3} B^{6}+\frac{27}{4} k_{3} g_{1}^{2} g_{3} B^{2}\right. \\
& \left.+3\left(\frac{9}{4}\right)^{2} k_{3} g_{3}^{2} g_{1} B^{4}\right)\left[\cos \left(2 \omega_{2}-\omega_{1}\right) t+\cos \left(2 \omega_{1}-\omega_{2}\right) t\right]
\end{aligned}
$$

and the expression of carrier signals of $V_{\text {out } T}(t)$ is

$$
\begin{aligned}
& V_{C}=\left(k_{1}+1\right)\left(g_{1} B+\frac{9}{4} g_{3} B^{3}\right)+\frac{3}{4} k_{3}\left(g_{1} B+\frac{9}{4} g_{3} B^{3}\right)^{3}(5) \\
& +\left(\frac{3}{4}\right)^{2} k_{3} g_{3} B^{3}\left(g_{1} B+\frac{9}{4} g_{3} B^{3}\right)^{2}\left(\cos \omega_{1} t+\cos \omega_{2} t\right)
\end{aligned}
$$

When designing a predistortion linearizer, two constructions should be followed: 1 . The circuit can generate IMD(this is why in some conditions the predistortion linearizer is called IMD generator); 2. The minimum cancellation of signals at carrier frequencies (this can bring less insertion loss). Based on these two principles and according to equation (3) and (4), we can get $g_{1}$ and $g_{2}$.

Fig. 2 shows the model of one predistortion linearizer. It is composed of a hybrid, whose input (1)and output(4) ports are the input and output ports of predistorter respectively. To barrier the DC current from bias of the diodes, DC blocks are utilized. The other two ports(2)、(3) of the hybrid which are $90^{\circ}$ out of phase connect the compensation circuits and comprises a pair of antiparallel diodes severing as nonlinear elements and impedance adjusting unites to adjust the impedance seen by the antiparallel diodes.

Supposed that the hybrid is working at its ideal type, according to its $\mathrm{S}$ parameter matrix, we have

$$
V_{\text {outP }}(t)=-j \Gamma V_{\text {iP }}(t)(6)
$$

where $\Gamma$ is the reflection coefficient seen from port (2)(or (3)) to the IMD generator. Here the antiparallel diodes are equal to a variable resistance, hence the impedance network and the bias voltage both influence complex resistance, and then change $\Gamma$. So to change the magnitude and phase of the IMD is to change the impedance network as well as the bias voltage. When designing a PD, first get the model parameters of the PD from the nonlinear characteristics of the TWTA according to equation (1) to (4). Second calculate the characteristic impedance and electrical angel of the microstrip stub serving part of the impedance network together with the load resistance. Then adjust the bias voltage to optimize the nonlinear characteristic produced by this PD. The advantage of this type of PD is that the I/O impedance characteristic would not be impacted by the nonlinear elements, and it has a small VSWR.

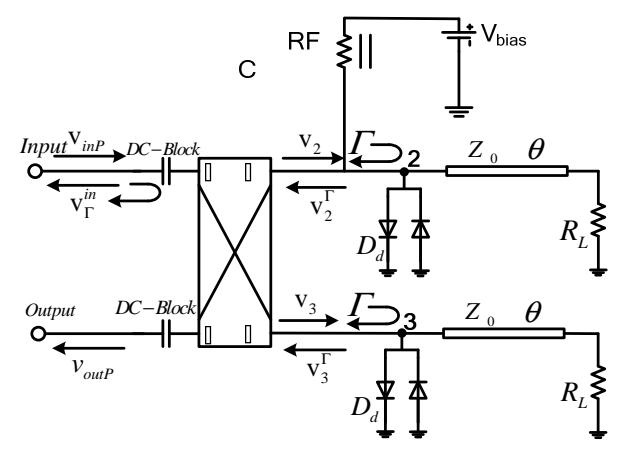

Fig. 2 the model of the predistortion circuit

\section{C. realization of the key elements of $P D$}

To realize the $\mathrm{PD}$, microstrip DC block to isolate DC signal, $90^{\circ}$ hybrid, microstrip bias circuit are needed. 


\section{1) microstrip $D C$ block}

Using a microstrip DC block to isolate DC signal is to simplify the whole structure, improve the stability, and minimize the parasitic parameters due to some capacitance elements. Interdigital coupled microstrip line can meet those needs within a broad bandwidth. Fig. 3(a) is a microstrip DC block whose equivalent circuit is a double series stubs Fig. 3(b). The [Z] matrix of the circuit in Fig. 3(b) is as follows, where $Z_{0 o}$ and $Z_{0 e}$ means odd and even characteristic resistance respectively. [S] matrix is then derived from equation (7), and its bandpass characteristic meets the needs as a microstrip DC block.

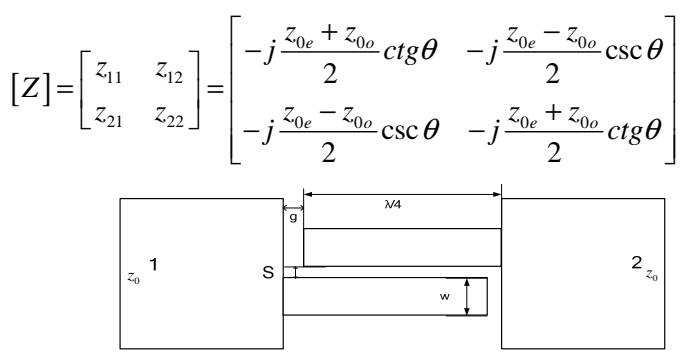

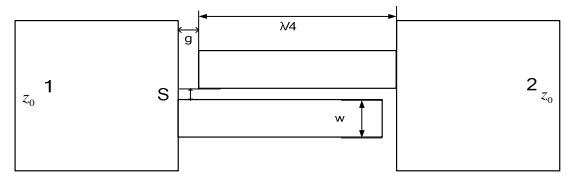

(a)

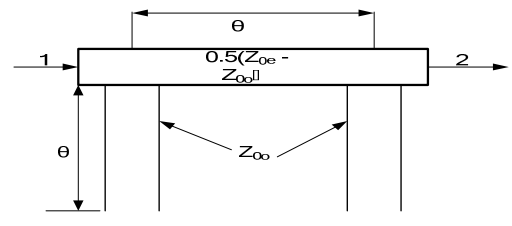

(b)

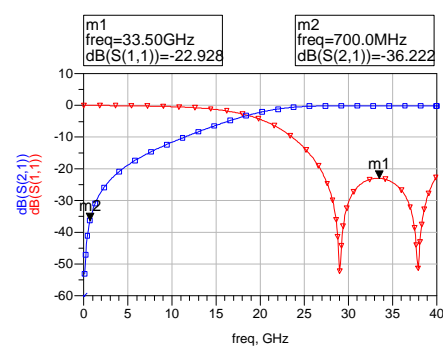

Fig . 3 microstrip DC block and simulation result

2) microstrip bias circuit

A microstrip sector bias is used to provide DC current for the diodes. Simulation in Advanced Design System (ADS) of Agilent shows that, radius $\mathrm{R}$ 、 angel $\theta$ and characteristic resistance $\mathrm{w}$ of the sector together with the electrical angel $\mathrm{L}_{1}$ of the high resistance line between the sector and the main transmission line are the key factors to be optimized.

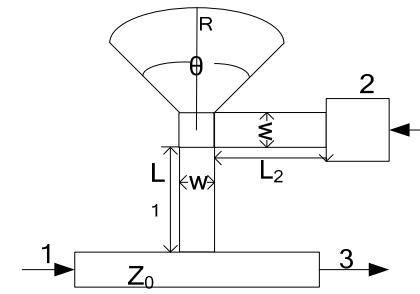

(a)

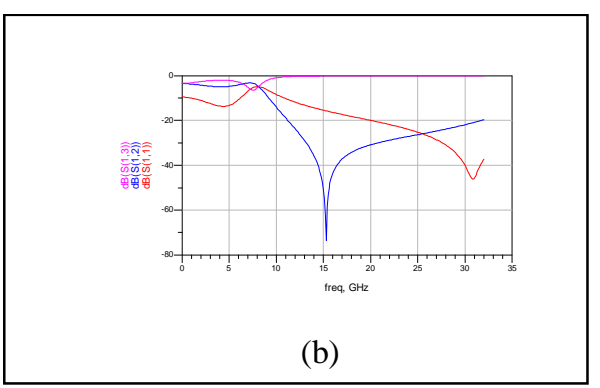

3) $90^{\circ}$ hybrid

Fig. 4 Bias circuit and its simulation results

$90^{\circ}$ hybrid (Fig. 5(a))is a kind of narrow-band coupler, and the characteristic resistance together with its electrical angel of the branch line are the key factors. Fig. 5(b) is the simulation results for the hybrid working at the frequency band of $29 \mathrm{GHz}$ and $31 \mathrm{GHz}$.

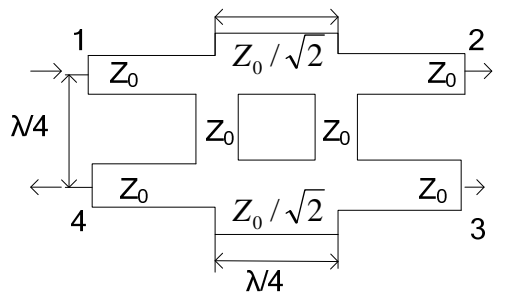

(a)

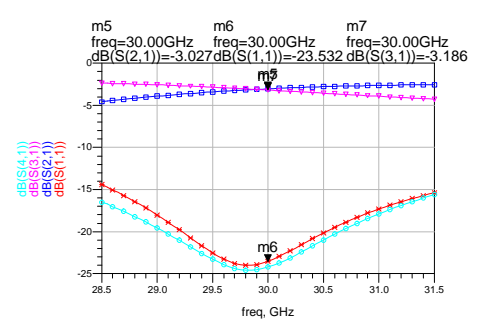

(b)

Fig. 5 quadrature hybrid and simulation results

\section{D. simulation design of the $P D$}

In order to validate the proposed predistortion linearizer circuit, system simulation is done. The substrate is Rogers 5880 with the dielectric constant of 2.2、 tan delta of 0.0009 and thickness of $0.254 \mathrm{~mm}$. The simulation results are illustrated in Fig.6 and Fig.7.

AM/AM and AM/PM characteristics of TWTA over a dynamic range of $26 \mathrm{~dB}$ (from its saturation point to backoff 
of $20 \mathrm{~dB}$ ) are $-5.6 \mathrm{~dB}$ and $-42^{\circ}$ respectively. Insertion loss of the $\mathrm{PD}$ together with the small signal drive amplifier and attenuator is $0 \mathrm{~dB}$ at linear region. And $\mathrm{AM} / \mathrm{AM}$ and $\mathrm{AM} / \mathrm{PM}$ characteristics of $\mathrm{PD}$ are $4.8 \mathrm{~dB}$ and $-32^{\circ}$ respectively.

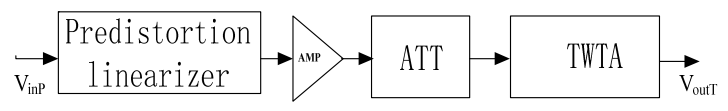

Fig. 6 Simulation line-up of linearized TWTA

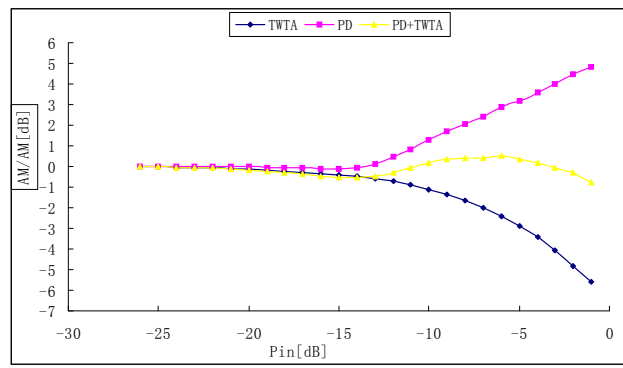

(a) AM/AM characteristic

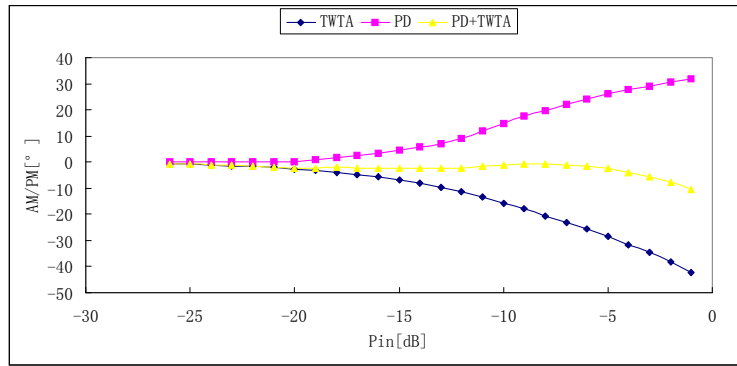

(b) $\mathrm{AM} / \mathrm{PM}$ characteristic

Fig. 7 Simulation results of linearized TWTA

\section{EXPERIMENT RESULTS}

Carrier to intermodulation distortion ratio(C/I) is a parameter used to characterize magnitude and phase distortion in amplifier systems. Here we use carrier to 3rd intermodulation distortion ratio(C/IM3) to show the nonlinear distortion of the linearized TWTA. Fig.8 is the measurement system and Fig.9 is the results. It is clear that, when cascaded with the proposed predistortion linearzer at the input port, C/IM3 of the TWTA (backoff 3dB from saturation) at the upper and lower sideband of central frequency is $27.04 \mathrm{dBc}$ and $27.89 \mathrm{dBc}$ respectively. That means the C/IM3 improvement of the TWTA with and without external linearizer is $13 \mathrm{~dB}$. The maximum improvement is $19 \mathrm{dBc}$ when backoff $6 \mathrm{~dB}$ from saturation.

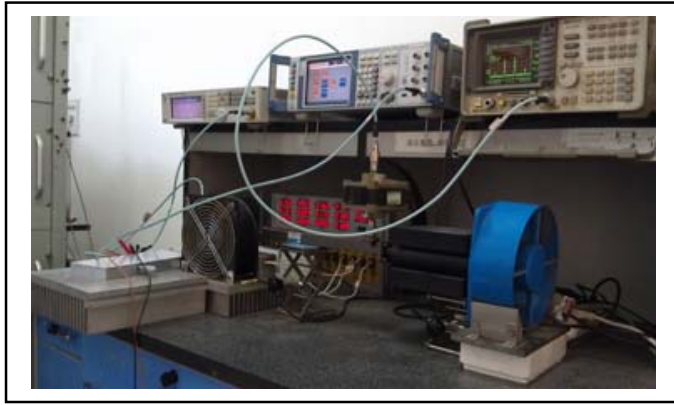

Fig. 8 measuring of the linearized TWTA

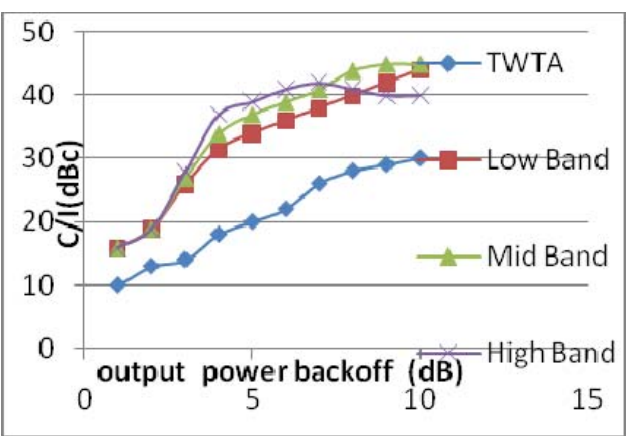

Fig. 9 test results of C/IM3

\section{CONCLUTIN}

Based on detailed analysis of nonlinear characteristics of TWTA and its predistortion linearization, a new linearizer working at the $\mathrm{Ka}$ band is proposed. This linearizer is composed of $90^{\circ}$ hybrid and nonlinear diodes together with microstrip bias as well as microstrip DC block. Cascated two-tone experiments of TWTA and proposed linearizer show that the design is effective. Compared with similar design[10], by tuning bias voltage, impedance stub and load resistance, this simple structure can flexibly modify the nonlinear characteristic, and greatly improve TWTA nonlinearity, and the realization is to some degree easy. Besides, conclusion from theoretical analysis can do some guide to similar design.

\section{REFERENCES}

[1] Weekley J and Mangus B. "TWTA versus SSPA: a comparison of onorbit data”. IEEE Transactions on Electron Devices, 2005, 52(5): 650-652.

[2] Katz A. "Linearization: Reducing distortion in power amplifiers ". IEEE Microwave Mag., 2001 ,2 (4): 37-49.

[3] Gray R, Katz A, and Dorval R. "Advances in millimeter-wave linearization”. Proceedings 13th Ka Broadband Communication Conference, Torino, Italy, 2007 (5): 76-79.

[4] Katz A, Gray R, and Dorval R. "Wide/multiband linearization of TWTAs using predistortion”. IEEE Transactions on Electron Devices, 2009, 56(5): 959-964.

[5] J.X. Qiu, D.K. Abe, B. Levush. "Linearizability of TWTAs Using Predistortion Techniques”. IEEE Transactions on Electron Devices, 2005,52 (5): 718-727

[6] Xin HU, Gang WANG, JiRun LUO. "Predistortion linearization of an X-band TWTA for Communications Applications”, IEEE Transactions on Electron Devices, 2011 ,58 (6): 1768-1774

[7] $\mathrm{Hu}$ Xin,Wang Gang,Wang Zi-cheng. "Improvement of TWT Nonlinearity with a Field Effect Transistor Predistortion Circuit”. 
Journal of Electronics \& Information Technology, 2011 ,33 (4): 951955

[8] W-M. Zhang, C. Yuen. "A Broadband Linearizer for Ka-Band Satellite Communication”, ARFTG conference Digest-Spring, Baltimore, MD, USA, 1998, 1998:33(1), 135-138.

[9] K.Yamauchi, K. Mori, M. Nakayama. "A microwave miniaturized linearizer using a parallel diode with a bias feed resistance ". IEEE Trans. Microwave Theory \& Tech., 1997 ,45 (12): 2431-2435.

[10] Song Jiaqian, Niu Zhongxia, Zhou Dongfang. "Study on Predistortion Technology Used in Linear TWTA". JOURNAL OF MICROWAVES, 2008 ,24 (5): 69-73 